\title{
microRNA-18a regulates gastric carcinoma cell apoptosis and invasion by suppressing hypoxia-inducible factor-1 $\alpha$ expression
}

\author{
FUBING WU*, WEN HUANG ${ }^{*}$ and XING WANG \\ Cancer Center, The First Hospital Affiliated to Fuzhou General Hospital of Nanjing Military Command of Chinese PLA, \\ Putian, Fujian 351100, P.R. China
}

Received August 17, 2014; Accepted May 1, 2015

DOI: 10.3892/etm.2015.2546

\begin{abstract}
Hypoxia is associated with various pathophysiological events, including cancer, lung and cardiovascular diseases. A number of studies have indicated that alterations in microRNA (miRNA) expression may be involved in the regulation of the cellular response to hypoxia. In the present study, miR-18a expression was revealed to be markedly downregulated under hypoxic conditions in MGC-803 and HGC-27 gastric carcinoma cell lines. Furthermore, miR-18a was demonstrated to affect the rate of cell apoptosis and the cell invasion ability in MGC-803 and HGC-27 cells under hypoxic conditions. Cell apoptosis was were analyzed using flow cytometry and cell invasiveness was evaluated using a Transwell-matrigel assay. The results showed that miR-18a overexpression was able to promote cell apoptosis and inhibit cell invasion. Using bioinformatic analysis, hypoxia-inducible factor (HIF)-1 $\alpha$ was identified as one of the target genes of miR-18a, and based on the function of HIF-1 $\alpha$ in hypoxia, miR-18a was predicted to regulate HIF-1 $\alpha$ expression. This hypothesis was confirmed by a further luciferase assay and the detection of the mRNA and protein expression levels of HIF-1 $\alpha$ following the induction of miR-18a overexpression. In addition, the expression levels of mitochondrial apoptosis-associated genes were detected following the induction of miR-18a overexpression. In the cells overexpressing miR-18a, the Bcl-2 protein expression level was downregulated, while the protein expression levels of Bax, caspase 3 and caspase 9 were upregulated in the MGC-803 and HGC-27 cell lines. Therefore, miR-18a was hypothesized to induce apoptosis through the HIF-1 $\alpha /$ mitochondrial apoptosis pathway.
\end{abstract}

Correspondence to: Professor Xing Wang, Cancer Center, The First Hospital Affiliated to Fuzhou General Hospital of Nanjing Military Command of Chinese PLA, 485 Dongyan Road, Putian, Fujian 351100, P.R. China

E-mail: wxing1972@126.com

*Contributed equally

Key words: microRNA-18a, gastric carcinoma, cell apoptosis, cell invasion, hypoxia-inducible factor- $1 \alpha$

\section{Introduction}

microRNAs (miRNAs) are non-coding RNA molecules, comprising $\sim 22$ nucleotides, that are evolutionarily conserved and function as sequence-specific regulators of gene expression through translational repression and/or transcript cleavage (1-4). miRNAs downregulate the protein expression of target genes through affecting their translation by binding to the 3'-untranslated regions (UTRs), or downregulate the mRNA expression of target genes by inducing the direct degradation of target mRNAs. Since the first miRNA, lin-4, was identified in Caenorhabditis elegans in 1993 (5), > 24,521 miRNAs have been identified, of which all are included in the miRBase database $(6,7)$. Previous studies have demonstrated that miRNAs play an important role in cell growth, differentiation, apoptosis, fat metabolism, morphogenesis and viral infection $(8,9)$. In recent years, an increasing number of studies have investigated the role of miRNAs in cancer. These studies have revealed that specific miRNAs exhibit an aberrant expression profile in tumor tissue, and are involved in various aspects of tumorigenesis, including growth, apoptosis, metastasis and particularly angiogenesis (10-14). Furthermore, a number of studies have indicated that alterations in miRNA expression may be involved in the regulation of the cellular response to hypoxia (15-18).

Hypoxia is associated with various pathophysiological events, including cancer, lung and cardiovascular diseases (19). In mammals, the hypoxia-dependent changes, on a gene expression level, are primarily mediated by the $\alpha$-subunits of hypoxia-inducible transcription factors (HIFs). HIF-1 $\alpha$ expression is maintained at lower level by proteasomal degradation during normoxia, while the degradation of HIF-1 $\alpha$ is inhibited under hypoxia (20). A previous study revealed that HIF-1 $\alpha$ was able to regulate the expression profile of a number of miRNAs, and certain miRNAs were also able to affect the protein expression level of HIF-1 $\alpha$ (21). For example, in the study by Cha et al, miR-519c was demonstrated to be a hypoxia-independent regulator of HIF-1 $\alpha$, functioning through the direct binding to the 3 '-UTR of the HIF-1 $\alpha$ gene and leading to reduced tumor angiogenesis (21).

The aim of the present study was to investigate miR-18a expression levels and the effect of miR-18a on cell apoptosis and invasion in gastric carcinoma cells subjected to hypoxic conditions. Furthermore, the target gene of miR-18a was identi- 
fied based on the results of a luciferase assay and the detection of mRNA and protein expression levels. Subsequently, the expression levels of apoptosis-associated genes were detected in the cell lines with miR-18a overexpression.

\section{Materials and methods}

Cell culture and hypoxia exposure. Human gastric carcinoma cell lines, MGC-803 and HGC-27, were purchased from the China Center for Type Culture Collection (Wuhan, China). MGC-803 and HGC-27 cells were cultured in RPMI 1640 media supplemented with $10 \%$ fetal bovine serum (HyClone, Logan, UT, USA). To expose the cells to hypoxia, the cells were cultured in a Billups-Rotenburg chamber (Billups-Rothenberg, Inc., Del Mar, CA, USA) with $94 \% \mathrm{~N}_{2}, 1 \% \mathrm{O}_{2}$ and $5 \% \mathrm{CO}_{2}$ at $37^{\circ} \mathrm{C}$ for a certain time.

miRNA mimics transient transfection. miR-18a mimics and negative control were purchased from Guangzhou RiboBio Co., Ltd. (Guangzhou, China). The cells were plated to $50 \%$ confluency and transfected with $200 \mathrm{nM}$ miR-18a mimics or negative control using Lipofectamine 2000 (Invitrogen Life Technologies, Carlsbad, CA, USA), according to the manufacturer's instructions. At 24 or $48 \mathrm{~h}$ after transfection, the cells were harvested for the further experiments.

Cell apoptosis assay. At $48 \mathrm{~h}$ after transfection, the cells were harvested and 500- $\mu 1$ samples were added to fluorescence-activated cell sorting tubes. The solutions were mixed with $25 \mathrm{ng} / \mathrm{ml}$ fluorescein isothiocyanate-labeled annexin $\mathrm{V}$ and $10 \mathrm{mg} / \mathrm{ml}$ propidium iodide, and incubated for $15 \mathrm{~min}$ at room temperature in the dark. Subsequently, the cells were analyzed by flow cytometry (BD Biosciences, San Jose, CA, USA).

Cell invasion. MGC-803 and HGC-27 cells were transfected with the miR-18a-5p mimics, HIF-1 $\alpha$ siRNA or negative control, cultivated for $24 \mathrm{~h}$ and transferred to the top of Matrigel-coated chambers (24-well insert; $8-\mu \mathrm{m}$ pore size; BD Biosciences) in serum-free RPMI 1640 medium. Medium containing $20 \%$ fetal calf serum was added to the lower chamber as a chemoattractant. Following incubation for $24 \mathrm{~h}$, the non-invaded cells were removed from the upper well with cotton swabs, while the invaded cells were fixed with $4 \%$ paraformaldehyde, stained with hematoxylin, and photographed (magnification, x200) in five independent fields for each well, using an optical microscope (Olympus Corporation, Tokyo, Japan). Each test was performed in triplicate.

Reverse transcription quantitative polymerase chain reaction (PCR). Total RNA was extracted using TRIzol reagent (Invitrogen Life Technologies), according to the manufacturer's instructions. For cDNA synthesis, $2 \mu \mathrm{g}$ total RNA was mixed with 500 ng oligo(dT) primers (Promega Corporation, Madison, WI, USA) or miRNA specific primers. The samples were incubated at $65^{\circ} \mathrm{C}$ for 10 min with $5 \mu 15 \mathrm{X}$ first-strand buffer, $2 \mu 1 \mathrm{dNTP}(5 \mathrm{mM}), 20$ units RNasin (Takara Bio, Inc., Otsu, Japan), $1 \mu 1 \mathrm{M}-\mathrm{MLV}$ reverse transcriptase (Promega Corporation) and distilled water to a total volume of $25 \mu 1$.
The quantitative PCR assay mixture contained $12.5 \mu 12 \mathrm{X}$ SYBR Green PCR Mix (Fermentas; Thermo Fisher Scientific), $0.3 \mu \mathrm{M}$ gene-specific forward and reverse primers, and $1 \mu \mathrm{l}$ cDNA template, which was made up to a final volume of $25 \mu 1$ with distilled water. Thermal cycling parameters were set as follows: Initial activation at $95^{\circ} \mathrm{C}$ for $5 \mathrm{~min}$, followed by 35 amplification cycles of denaturation at $94^{\circ} \mathrm{C}$ for $30 \mathrm{sec}$, annealing at $58^{\circ} \mathrm{C}$ for $30 \mathrm{sec}$ and extension at $72^{\circ} \mathrm{C}$ for $15 \mathrm{sec}$. The levels of gene expression were calculated by relative quantification against GAPDH or U6 snRNA, which were used as the internal controls, with U6 snRNA used as an internal reference gene for the qPCR detection of MiR-18a. All samples were amplified in triplicate, and the data analysis was conducted using MxPro qPCR system software (Stratagene, La Jolla, CA, USA).

Western blot analysis. MGC-803 and HGC-27 cells $\left(2 \times 10^{6}\right)$ were collected and washed twice with ice-cold phosphate-buffered saline (PBS). The cell pellets were suspended in radioimmunoprecipitation assay lysis buffer for $30 \mathrm{~min}$ on ice, after which the lysates were centrifuged at $12,000 \mathrm{x} \mathrm{g}$ at $4^{\circ} \mathrm{C}$ for $20 \mathrm{~min}$. Equal amounts of protein in the supernatant were isolated by $12 \%$ SDS polyacrylamide gel electrophoresis and transferred onto a polyvinylidene difluoride membrane (Millipore Corporation, Billerica, MA, USA). The membranes were blocked for $1 \mathrm{~h}$ at $37^{\circ} \mathrm{C}$ with $5 \%$ non-fat milk, and subsequently incubated with mouse monoclonal anti-HIF-1 $\alpha$ (1:500; \#359702, BioLegend, Inc., San Diego, CA, USA), anti-Bax (1:1,000; Abcam, Cambridge, MA, USA), Bcl-2 (1:1,500; \#ab117115, Abcam), caspase 3 (1:1,000; \#ab2171, Abcam), caspase 9 (1:1,000; \#ab28131, Abcam) and GAPDH (1:400; \#sc-365062, Santa Cruz Biotechnology, Inc., Santa Cruz, CA, USA) antibodies in 5\% non-fat milk for $1 \mathrm{~h}$ at $37^{\circ} \mathrm{C}$. After washing in PBS with $0.5 \%$ Tween 20 (PBST), the membrane was incubated with a rabbit anti-mouse horseradish peroxidase-conjugated secondary antibody (1:4,000; \#ab6728, Abcam) at room temperature for $1 \mathrm{~h}$. After further washing with PBST, the membrane was assayed with enhanced chemiluminescence with a chemiluminescence HRP subtrate (Millipore Corporation) and visualized on X-ray films.

Vector construction and luciferase reporter assay. To construct a luciferase reporter vector, the wild-type 3'-UTR or the mutant 3'-UTR of HIF-1 $\alpha$, which contained putative binding sites for miR-18a-5p, were subcloned into the psiCHECK-2 vector (Promega Corporation). MGC-803 cells were plated at a density of $5 \times 10^{4}$ cells per well in 24-well plates. The following day, psiCHECK-2 luciferase vectors, which included the 3'-UTR of HIF-1 $\alpha$ and miR-18a-5p mimics or negative control oligonucleotides, were transfected into the cells using Lipofectamine 2000. At $48 \mathrm{~h}$ after transfection, the luciferase assays were performed using a dual luciferase reporter assay system (Promega Corporation).

Statistical analysis. Statistical analysis was performed using the SPSS 19.0 software package (IBM SPSS, Armonk, NY, USA). All numerical data were analyzed using the Student's $\mathrm{t}$-test, and all the tests performed were two-sided. $\mathrm{P}<0.05$ was considered to indicate a statistically significant difference. 


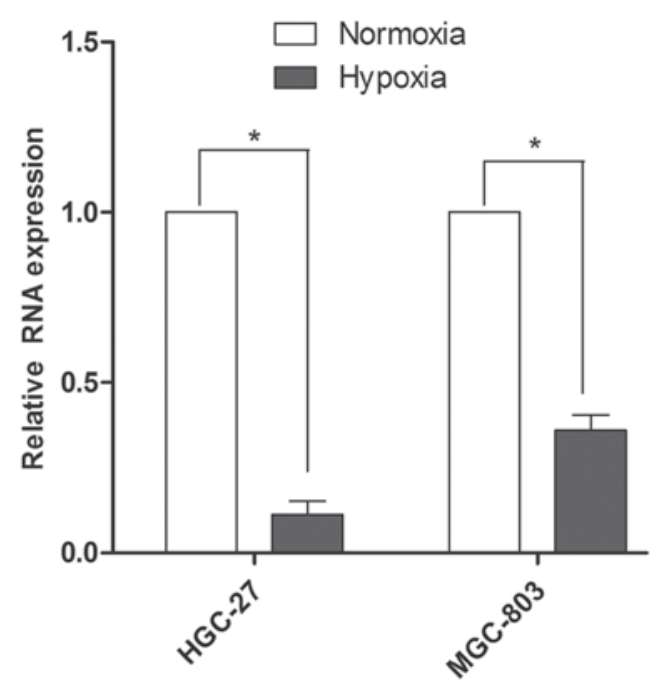

Figure 1. Expression level of miR-18a in MGC-803 and HGC-27 cells under hypoxic conditions. Cells were cultured in $94 \% \mathrm{~N}_{2}, 1 \% \mathrm{O}_{2}$ and $5 \% \mathrm{CO}_{2}$ at $37^{\circ} \mathrm{C}$ for $12 \mathrm{~h}$. The histogram data are expressed as the mean \pm standard deviation. ${ }^{*} \mathrm{P}<0.05$.

\section{Results}

Hypoxia downregulates the expression of $m i R-18 a$. To investigate the function of miR-18a in gastric carcinoma cells under hypoxic conditions, the expression level of miR-18a in MGC-803 and HGC-27 cell lines was initially detected. As shown in Fig. 1, the expression level of miR-18a was downregulated in the two cell lines subjected to hypoxic conditions. In the HGC-27 cells, the miR-18a expression level under hypoxic conditions was $89 \%$ lower compared with the expression under normoxic conditions. Furthermore, in the MGC-803 cell line, the miR-18a expression level under hypoxic conditions was $64 \%$ lower compared with the expression under normoxic conditions.

miR-18a regulates cell apoptosis under hypoxic conditions. The apoptosis rate of the gastric carcinoma cells was investigated using flow cytometry following subjection to hypoxic conditions for $24 \mathrm{~h}$. As shown in Fig. 2A, miR-18a markedly affected the rate of apoptosis in the HGC-27 and MGC-803 cell lines. Early and late apoptosis rates (lower right and upper right quadrants of the FITC graphs, respectively; Fig. 2A) for the HGC-27 cells were 5.8 and $4.9 \%$, respectively, transfected with negative control (NC) mimics. In addition, early and late apoptosis rates for the MGC-803 cells were 9.6 and $2.9 \%$, respectively, transfected with NC mimics. By contrast, in the cells overexpressing miR-18a subjected to hypoxic conditions, the early and late apoptosis rates of HGC-27 cells were 23.9 and $8.0 \%$, respectively. In addition, the early and late apoptosis rates of the MGC-803 cells were 29.3 and $13.6 \%$, respectively, in the cells subjected to hypoxic conditions and miR-18a overexpression. As shown in Fig. 2B, the total apoptosis rates of HGC-27 and MGC-803 cells increased 2.98-fold and 3.43-fold following miR-18a mimics transfection, respectively.

miR-18a regulates the invasion capacity of cells under hypoxic conditions. The invasion ability of gastric carcinoma
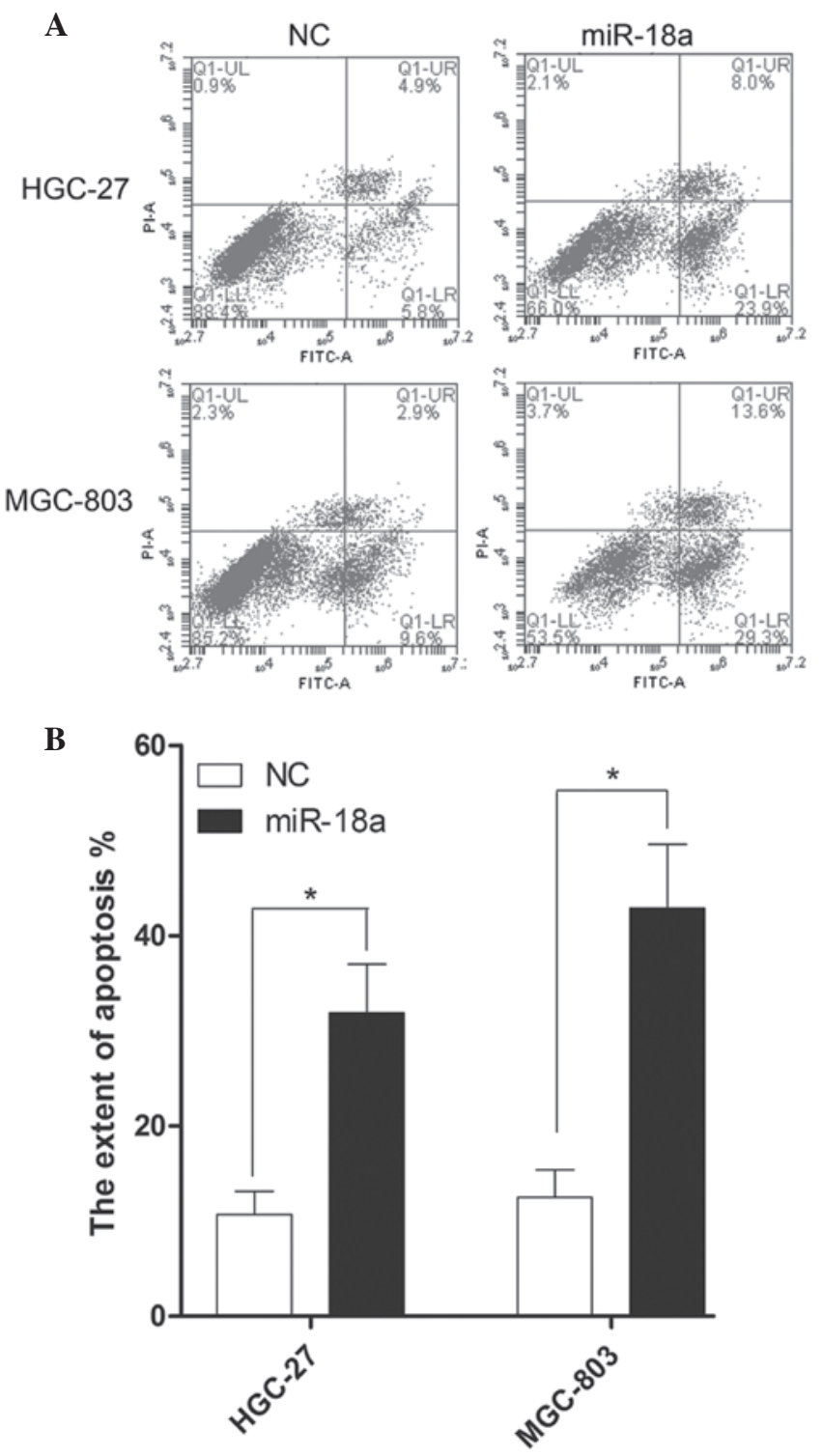

Figure 2. Overexpression of miR-18a increases the extent of apoptosis in the MGC-803 and HGC-27 cell lines subjected to hypoxic conditions. (A) Flow cytometry results with annexin-FITC/PI staining. Cells were transfected with miR-18a mimics or NC mimics. Following hypoxia for $24 \mathrm{~h}$, the cells were harvested and the rate of apoptosis was analyzed by flow cytometry. (B) Extent of apoptosis among the different experimental groups. The apoptosis ratio was calculated as the early apoptosis (lower right quadrant) plus the late apoptosis (upper right quadrant) percentage. Data are presented as the mean \pm standard deviation. ${ }^{*} \mathrm{P}<0.05$. FITC, fluorescein isothiocyanate; $\mathrm{miR}$, microRNA; NC, negative control; PI, propidium iodide.

cells was investigated using a Transwell assay following the induction of hypoxic conditions for $24 \mathrm{~h}$. As shown in Fig. 3A, miR-18a markedly affected the invasion ability of the HGC-27 and MGC-803 cells. The numbers of HGC-27 cells that had invaded the chamber following transfection with the negative control mimics or the miR-18a mimics were $152 \pm 8$ and $49 \pm 5$, respectively. Furthermore, the numbers of invasive MGC-803 cells following transfection with the negative control or miR-18a mimics were $165 \pm 7$ and $42 \pm 4$, respectively. As shown in Fig. 3B, the total number of invasive cells in the HGC-27 and MGC-803 cell lines decreased by 66.76 and $74.55 \%$ following miR-18a mimics transfection, respectively. 

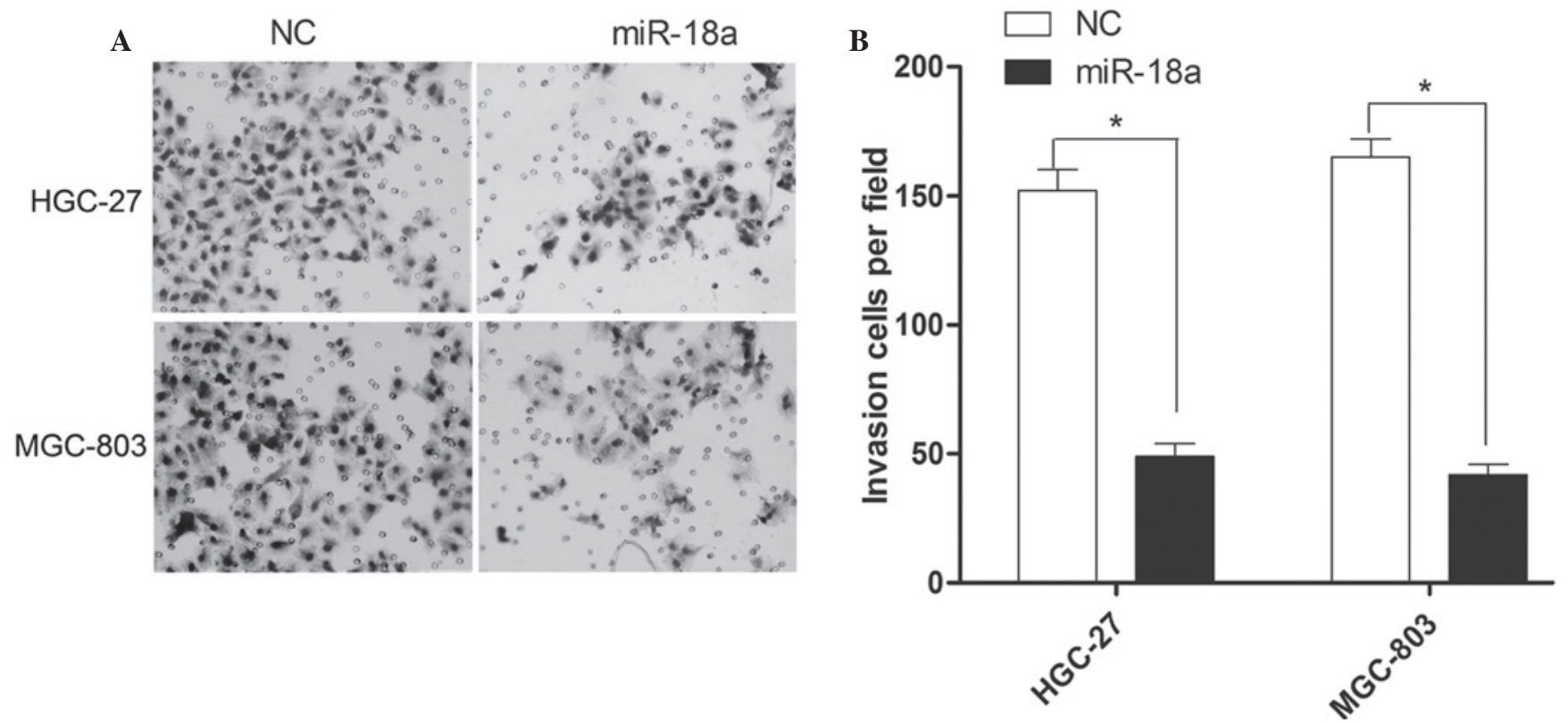

Figure 3. Effect of miR-18a overexpression on cell invasion under hypoxic conditions. (A) Representative images showing the invasive ability of the MGC-803 and HGC-27 cell lines in each group. The invasion ability of the MGC-803 and HGC-27 cells was detected using a Matrigel invasion assay at $24 \mathrm{~h}$ after the transfection of miR-18a mimics or NC mimics. (B) Average number of invasive cells per field among the different experimental groups. Data are presented as the mean \pm standard deviation. ${ }^{*} \mathrm{P}<0.05$. NC, negative control; miR, microRNA.

A

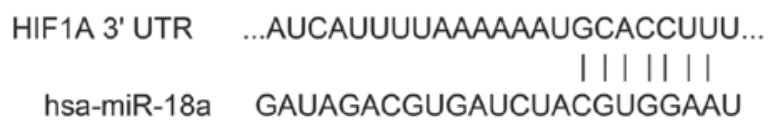

C

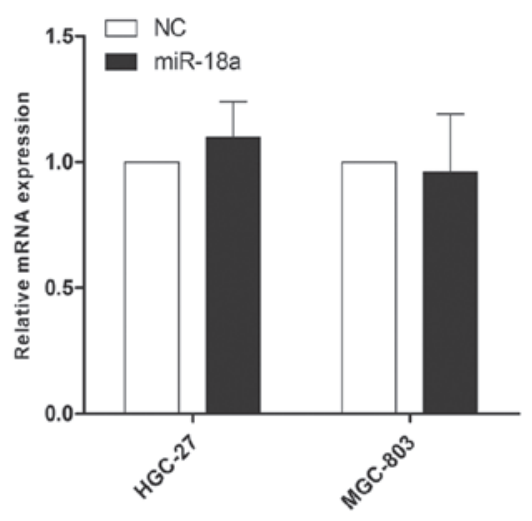

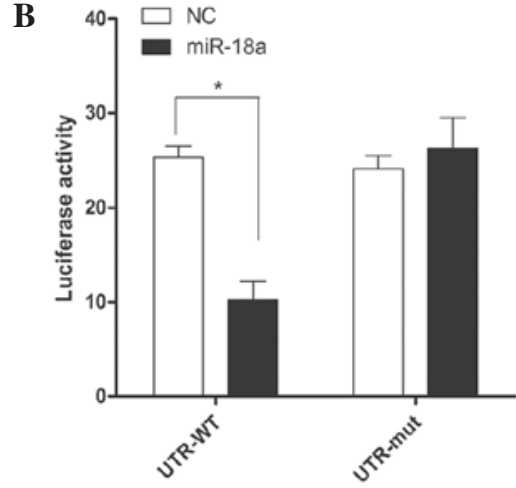

D

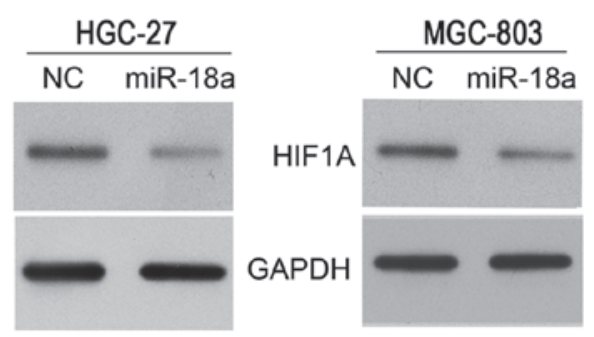

Figure 4. HIF-1 $\alpha$ is a direct target of miR-18a. (A) Predicted duplex formation between the 3'-UTR of HIF-1 $\alpha$ and miR-18a. (B) Luciferase activity following the incorporation of the wild-type (UTR-WT) or mutant (UTR-mut) HIF-1 $\alpha$ 3'-UTR reporter gene in the MGC-803 cells transfected with miR-18a or NC mimics. (C) Quantitative polymerase chain reaction analysis showing the mRNA expression levels of HIF-1 $\alpha$ in the MGC-803 and HGC-27 cell lines transfected with the miR-18a or NC mimics. Data were normalized against the mRNA expression level of GAPDH. (D) Western blot analysis showing the protein expression levels of HIF-1 $\alpha$ in the MGC-803 and HGC-27 cell lines transfected with the miR-18a or NC mimics. "P<0.05. HIF, hypoxia-inducible factor; UTR, untranslated region; miR, microRNA; NC, negative control.

$H I F-1 \alpha$ is a direct target of miR-18a. To understand the mechanisms through which miR-18a induces cell apoptosis and inhibits tumor invasion, several computational methods were used to identify miR-18a targets in humans, and the predicted duplex formation between the 3'-UTR of HIF-1a and miR-18a is shown in Fig. 4A. Among the 275 targets predicted using the TargetScan 6.2 software program,
HIF- $1 \alpha$ had been previously implicated in the regulation of cell apoptosis and/or invasion, which was of particular interest since the expression of HIF-1 $\alpha$ may be induced under hypoxic conditions. To investigate whether HIF-1 $\alpha$ was a direct target of miR-18a, a luciferase reporter assay was conducted. The results revealed that miR-18a inhibited the activity of the firefly luciferase with the wild-type $3^{\prime}$-UTR 


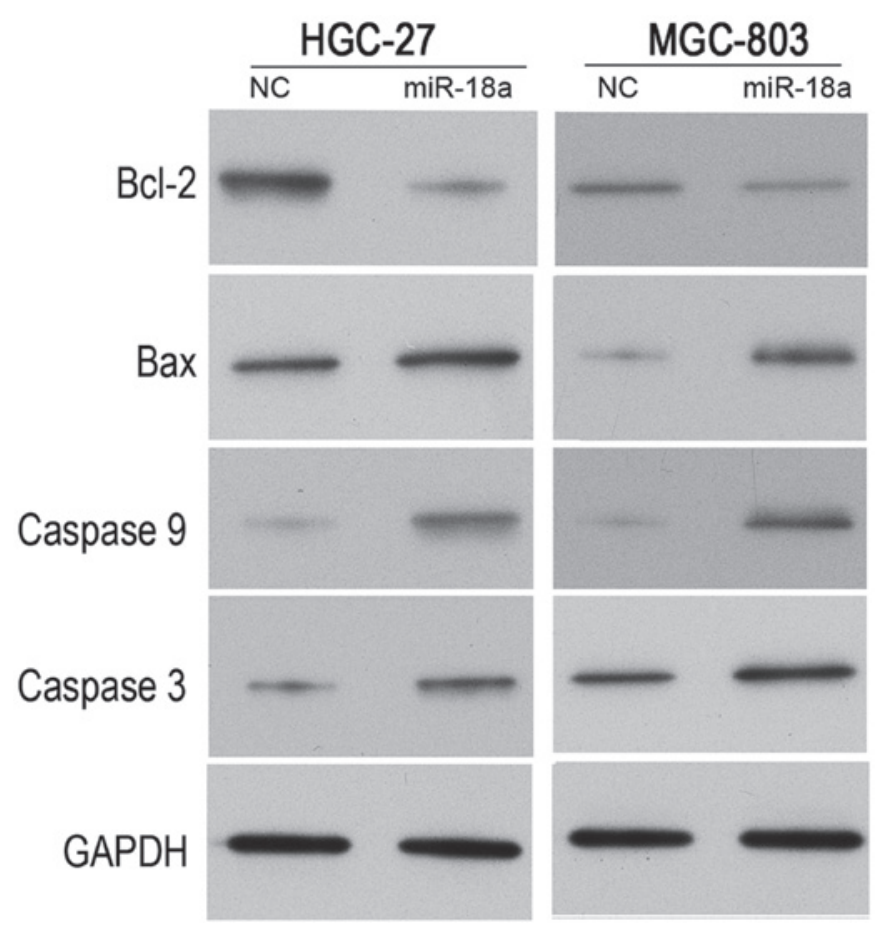

Figure 5. Protein expression levels of Bcl-2, Bax, caspase 3 and caspase 9 in the MGC-803 and HGC-27 cell lines subjected to hypoxic conditions in each group following transfection with miR-18a or NC mimics. NC, negative control; miR, microRNA.

of HIF-1 $\alpha$. By contrast, miR-18a exhibited no effect on the activity of the firefly luciferase with mutant type 3'-UTR of HIF-1 $\alpha$ (Fig. 4B). Subsequently, the effect of miR-18a overexpression on the mRNA and protein expression levels of HIF-1 $\alpha$ were investigated. Although miR-18a overexpression did not result in the degradation of HIF-1 $\alpha$ mRNA (Fig. 4C), miR-18a overexpression did reduce the activity of the luciferase reporter gene fused to the wild-type HIF-1 $\alpha$ 3'-UTR, indicating that miR-18a targets HIF-1 $\alpha$ through translational inhibition. In accordance with these results, a clear reduction in the level of endogenous HIF- $1 \alpha$ protein expression was observed in the MGC-803 and HGC-27 cells overexpressing miR-18 (Fig. 4D).

miR-18a regulates the expression of Bax, $\mathrm{Bcl}-2$, caspase 3 and caspase 9 under hypoxic conditions. To investigate the possible mechanisms underlying the effects of miR-18a on cell apoptosis, the implication of miR-18a in a typical signaling pathway of apoptosis was evaluated. Thus, the protein expression levels of Bcl-2, Bax, caspase 3 and caspase 9 were detected in cells subjected to hypoxic conditions following transfection with the miR-18a mimics or negative control mimics. As shown in Fig. 5, Bcl-2 protein expression levels were downregulated following the induction of miR-18a overexpression, while Bax protein expression levels were upregulated in the MGC-803 and HGC-27 cells. In addition, two important genes in the apoptosis signaling pathway were analyzed. The protein expression levels of caspase 3 and caspase 9 were demonstrated to be upregulated following the induction of miR-18a overexpression. These results indicated that the apoptosis signaling pathway may be activated by miR-18a under hypoxic conditions.

\section{Discussion}

In present study, miR-18a was initially found to be downregulated in the MGC-803 and HGC-27 gastric carcinoma cell lines subjected to hypoxic conditions. Hypoxia is an essential feature of a tumorous microenvironment. When cancer cells are placed in hypoxic conditions, a variety of signaling pathways that regulate proliferation, angiogenesis and cell death become activated. However, cancer cells have adapted to these pathways, allowing tumors to survive and even grow under hypoxic conditions (22). Thus, the identification of key factors that are able to inhibit the adaptation of cancer cells to hypoxic conditions is important. Previous studies have revealed that alterations in miRNA expression levels may be involved in the regulation of the cellular response to hypoxia (15-18). For example, the expression levels of miR-210 and miR-373 have been shown to be induced by HIF-1 $\alpha$, while miR-20b, miR-199a and miR-424 have been demonstrated to regulate the expression profile of HIF-1 $\alpha$ under hypoxic conditions (16). In the present study, the expression of miR-18a markedly changed under hypoxic conditions in the gastric carcinoma cells; thus, miR-18a was hypothesized to play a role in the regulation of gastric carcinoma cell behavior. In addition, the results demonstrated that miR-18a overexpression was able to inhibit the cell invasion ability and promote cell apoptosis in MGC-803 and HGC-27 cells subjected to hypoxic conditions. To the best of our knowledge, the present study is the first to report that miR-18a is able to regulate cell invasion and apoptosis under hypoxic conditions.

miRNAs exert a regulatory role on target genes. Through bioinformatics analysis, HIF-1 $\alpha$ was identified as a possible target gene of miR-18a.Subsequently, the results of the luciferase assay, quantitative PCR and western blot analysis confirmed that HIF-1 $\alpha$ was an indeed a target gene of miR-18a. HIF-1 $\alpha$ is an oxygen-dependent transcriptional activator. HIF-1 induces the transcription of $>60$ proteins, and these proteins are able to increase oxygen availability by promoting erythropoiesis and angiogenesis, which activates genes involved in glucose transport and metabolism $(23,24)$. Based on the important role of miR-18a in hypoxic conditions and the association between miR-18a and HIF-1 $\alpha$, miR-18a was hypothesized to affect cell invasion and apoptosis through HIF-1 $\alpha$.

To further investigate the mechanism underlying the regulation of cell apoptosis by miR-18a, the expression levels of apoptosis-associated genes were detected. Following the induction of miR-18a overexpression, Bcl-2 protein expression levels were downregulated, while the protein expression levels of Bax, caspase 3 and caspase 9 were upregulated in the MGC-803 and HGC-27 cell lines. These results demonstrated that miR-18a overexpression was able to activate the mitochondrial apoptosis pathway $(25,26)$. Thus, miR-18a was hypothesized to induce apoptosis through the HIF-1 $\alpha /$ mitochondrial apoptosis pathway.

However, the present study had a number of potential limitations. The results demonstrated that miR-18a inhibited apoptosis through the HIF-1 $\alpha /$ mitochondrial apoptosis pathway. However, other potential regulatory pathways between HIF-1 $\alpha$ and the mitochondrial apoptosis pathway were not assessed in the present study. In addition, the mechanism underlying miR-18a-induced regulation of cell invasion 
was not investigated. Therefore, future studies should focus their attention on solving these limitations.

In conclusion, the results of the present study indicated that miR-18a was downregulated and was able to affect cell apoptosis and invasion in MGC-803 and HGC-27 cell lines under hypoxic conditions. Furthermore, HIF- $1 \alpha$ was identified as a potential target gene of miR-18a. In addition, miR-18a overexpression was able to activate the mitochondrial apoptosis pathway. Thus, it is hypothesized that miR-18a may induce apoptosis via the HIF-1 $\alpha /$ mitochondrial apoptosis pathway.

\section{Acknowledgements}

This study was supported by a grant from the Science and Technology Planning Project of Putian City [no. 2012S02 (1)].

\section{References}

1. Zhang J, Du YY, Lin YF, Chen YT, Yang L, Wang HJ and Ma D The cell growth suppressor, miR-126, targets IRS-1. Biochem Biophys Res Commun 377: 136-140, 2008.

2. Luzi E, Marini F, Sala SC, Tognarini I, Galli G and Brandi ML: Osteogenic differentiation of human adipose tissue-derived stem cells is modulated by the miR-26a targeting of the SMAD1 transcription factor. J Bone Miner Res 23: 287-295, 2008.

3. Yu B, Chapman EJ, Yang Z, Carrington JC and Chen X: Transgenically expressed viral RNA silencing suppressors interfere with microRNA methylation in Arabidopsis. FEBS Lett 580: 3117-3120, 2006.

4. Bartel DP: MicroRNAs: Genomics, biogenesis, mechanism and function. Cell 116: 281-297, 2004.

5. Lee RC, Feinbaum RL and Ambros V: The C. elegans heterochronic gene lin-4 encodes small RNAs with antisense complementarity to lin-14. Cell 75: 843-854, 1993.

6. Griffiths-Jones S, Saini HK, van Dongen S and Enright AJ: miRBase: Tools for microRNA genomics. Nucleic Acids Res 36 (Database issue): D154-D158, 2008.

7. Kozomara A and Griffiths-Jones S: miRBase: annotating high confidence microRNAs using deep sequencing data. Nucleic Acids Res 42 (Database issue): D68-D73, 2014.

8. Giraldez AJ, Cinalli RM, Glasner ME, et al: MicroRNAs regulate brain morphogenesis in zebrafish. Science 308: 833-838, 2005.
9. Guo H, Ingolia NT, Weissman JS and Bartel DP: Mammalian microRNAs predominantly act to decrease target mRNA levels. Nature 466: 835-840, 2010.

10. Tsai WC, Hsu PW, Lai TC, et al: MicroRNA-122, a tumor suppressor microRNA that regulates intrahepatic metastasis of hepatocellular carcinoma. Hepatology 49: 1571-1582, 2009.

11. Xiong Y, Fang JH, Yun JP, Yang J, Zhang Y, Jia WH and Zhuang SM: Effects of microRNA-29 on apoptosis, tumorigenicity and prognosis of hepatocellular carcinoma. Hepatology 51: 836-845, 2010.

12. Xu T, Zhu Y, Xiong Y, Ge YY, Yun JP and Zhuang SM: MicroRNA-195 suppresses tumorigenicity and regulates G1/S transition of human hepatocellular carcinoma cells. Hepatology 50: 113-121, 2009.

13. Anand S: A brief primer on microRNAs and their roles in angiogenesis. Vasc Cell 5: 2, 2013.

14. Seok JK, Lee SH, Kim MJ and Lee YM: MicroRNA-382 induced by HIF-1 $\alpha$ is an angiogenic miR targeting the tumor suppressor phosphatase and tensin homolog. Nucleic Acids Res 42: 8062-8072, 2014.

15. Chan SY and Loscalzo J: MicroRNA-210: A unique and pleiotropic hypoxamir. Cell Cycle 9: 1072-1083, 2010.

16. Loscalzo J: The cellular response to hypoxia: Tuning the system with microRNAs. J Clin Invest 120: 3815-3817, 2010.

17. Kulshreshtha R, Ferracin M, Wojcik SE, et al: A microRNA signature of hypoxia. Mol Cell Biol 27: 1859-1867, 2007.

18. Kulshreshtha R, Davuluri RV, Calin GA and Ivan M: A microRNA component of the hypoxic response. Cell Death Differ 15: 667-671, 2008.

19. Adams JM, Difazio LT, Rolandelli RH, et al: HIF-1: A key mediator in hypoxia. Acta Physiol Hung 96: 19-28, 2009.

20. Huang LE, Gu J, Schau M and Bunn HF: Regulation of hypoxia-inducible factor 1alpha is mediated by an $\mathrm{O}_{2}$-dependent degradation domain via the ubiquitin-proteasome pathway. Proc Natl Acad Sci USA 95: 7987-7992, 1998.

21. Cha ST, Chen PS, Johansson G, et al: MicroRNA-519c suppresses hypoxia-inducible factor-1alpha expression and tumor angiogenesis. Cancer Res 70: 2675-2685, 2010.

22. Harris AL: Hypoxia - a key regulatory factor in tumour growth. Nat Rev Cancer 2: 38-47, 2002.

23. Semenza GL: HIF-1 and tumor progression: Pathophysiology and therapeutics. Trends Mol Med 8 (Suppl 4): 62-67, 2002.

24. Semenza GL: Targeting HIF-1 for cancer therapy. Nat Rev Cancer 3: 721-732, 2003.

25. Eskes R, Desagher S, Antonsson B and Martinou JC: Bid induces the oligomerization and insertion of Bax into the outermitochondrial membrane. Mol Cell Biol 20: 929-935, 2000.

26. Miramar MD, Costantini P, Ravagnan L, et al: NADH oxidase activity of mitochondrial apoptosis-inducing factor. J Biol Chem 276: 16391-16398, 2001. 